This is a postprint version of the following published document:

González, V. J., Martín-Alberca, C., Montalvo, G., García-Ruiz, C., Baselga, J., Terrones, M. \& Martin, O. (2014). Carbon nanotube-Cu hybrids enhanced catalytic activity in aqueous media. Carbon, 78, pp. 10-18.

DOI: $10.1016 /$ j.carbon.2014.06.014

(C) Elsevier, 2014

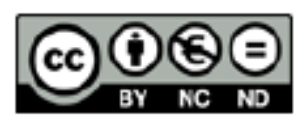

This work is licensed under a Creative Commons Attribution-NonCommercialNoDerivatives 4.0 International License. 


\title{
Carbon nanotube-Cu hybrids enhanced catalytic activity in aqueous media
}

\author{
Viviana JehováGonzález ${ }^{a}$, Carlos Martín-Alberca ${ }^{b, c}$, Gemma Montalvo ${ }^{b, c}$, Carmen García- \\ Ruiz $^{b, c}$, Juan Baselga ${ }^{a}$, Mauricio Terrones ${ }^{d}$, Olga Martin ${ }^{a, *}$
}

a Department of Materials Science and Engineering \& Chemical Engineering, Polytechnic School, Carlos III University of Madrid, Avenida Universidad 30, 28911 Leganés, Madrid, Spain

$\mathrm{b}$ Department of Analytical Chemistry, Physical Chemistry and Chemical Engineering, University of Alcalá, Carretera Madrid-Barcelona Km $33.600,28871$ Alcalá de Henares, Madrid, Spain

C University Institute of Research in Police Sciences (IUICP), University of Alcalá, Ctra. Madrid-Barcelona Km 33.600, 28871 Alcalá de Henares, Madrid, Spain

$\mathrm{d}$ Department of Physics, Department of Materials Science and Engineering, and Materials Research Institute, The Pennsylvania State University, 104 Davey Lab, University Park, PA 16802, United States

** Corresponding author: Fax: +34 9162499 40/30.

mail address: martinc@ing.uc3m.es (O. Martin)

In this work, we report the synthesis of a hybrid material, consisting of Cu nanoparticles (Cu-NPs) anchored on the surface of carbon nanotubes (CNTs), which is able to increase the catalytic behavior

when compared to bare CuNPs. This enhanced activity was evalu-ated by the electrophoretic separation of three carbohydrates: sucrose, glucose and fruc-tose. CNTs were first synthesized in the presence of minute amounts of oxygen, oxidized using $\mathrm{H}_{2} \mathrm{O}_{2}-\mathrm{UV}$ radiation and then decorated with

Cu-NPs. The resulting hybrid structure was confirmed and characterized by X-ray photoelectron spectroscopy, transmission elec-tron microscopy, high resolution transmission electron energydispersive X-ray spectros-copy, titration, thermogravimetric analysis, elemental analysis and Raman spectroscopy.

\section{Introduction}

Multi-walled carbon nanotubes (MWCNTs) were first imaged and their structure subsequently identified by Endo and Iijima, respectively $[1,2]$. Soon after, single-walled carbon nanotubes (SWCNTs) were synthesized and characterized [3]. Since then, carbon nanotubes (CNTs) attracted the attention of numerous scientists due to their fascinating electronic, mechanical, thermal, and electrochemical properties [4-6]. In order to modify the electronic properties of CNTs and induce surface reactivity, doping with $\mathrm{B}, \mathrm{N}, \mathrm{BN}, \mathrm{Si}$, P could be achieved [7]. Basically, doping results in an excess or deficiency of electrons which could be then used to fabricate electronic devices $[7,8]$.

Defect free and undoped CNTs are chemically inert and due to their large size (micrometers in length), CNTs are not readily dispersible in solvents. This fact makes difficult some applications needing uniform CNT dispersions in aqueous media. However, CNTs can be dispersed in water by a single step synthesis-functionalization with oxygen-derivatives [9]. In that case, oxygen-derivatives concentrate on the CNT surface and create the electrostatic stability required for creating 
a uniform colloidal dispersion [10]. Furthermore, the combination of two nanomaterials such as CNTs and Cu-NPs could result in a novel hybrid material useful in the fabrication of sensors or catalysts $[11,12]$. Examples of catalytic applications can be found with Pd- and Pt-NPs anchored to CNTs [13-17], and alloys of Pt-Sn [18], Pt-Co [19], and $\mathrm{Fe}_{3} \mathrm{O}_{4}-\mathrm{Pt}$ [20]. More recently, using a polymer assisted route, a variety of metal NPs (e.g. Au, Ag, Pd and Pt) were attached to the surface of CNTs. Interestingly, these materials exhibited a significantly enhanced catalytic activity in the reduction of 4-nitrophenol to produce 4-aminophenol [21]. In this context, the modification of CNTs with Cu-NPs or its oxides [22-24] also becomes an attractive possibility to use these hybrid materials as nanoelectronic devices [23] or as novel catalysts when used as electrodes for the electrochemical sensing of carbohy-drates [22,25-27].

In a variety of methods based on capillary electrophoresis (CE), oxidized CNTs were added to the background electrolyte (BGE) in order to enhance the resolution of electrophoretic separation [28]. However, for the detection of carbohydrates, CNTs were preferably used as a matrix so that $\mathrm{Cu}-\mathrm{NPs}$ could be deposited on the outer surface of CNTs [22,27] or graphene $[25,29]$. It is noteworthy that the literature is very scarce on topics related to applications of metal NPs-CNTs hybrid materials in aqueous media. One reason could be due to the fact that CNTs tend to aggregate and metals are also insoluble (dispersible) in water. Therefore, having uniform dispersions of CNTs is a real challenge which must be overcome when dealing with analytical applications in aqueous media. Overall, the high surface-to-volume ratio of NPs, along with the ability to control the particle size, provides a unique opportunity for developing further catalytic applications.

In order to anchor NPs on the surface of CNTs, different methods such as the reduction of metal salts [30-33], deposition of NPs on CNTs by thermal evaporation [23] or using hyperbranched polymers [21] can be used. Specifically, for the deposition of Cu-NPs on CNTs, numerous authors have reported different approaches including calcination treatments [24], chemical reduction [22], and microwave radiation [34]. In this paper, we first report on the Cu-NPs encapsulation over the CNTs surface using microwave radiation (MW), a method which appears to be a very efficient when compared to conventional methods [35]. It is known that CNT reach very high temperatures and weld or reconstruct under short MW pulses [36]. The presence of impurities such as Fe (catalyst residues) or even amorphous carbon may also contribute to an intense heating (Joule effect).

In this paper, we report the CNT functionalization with carboxylic oxygen-derivatives, and the simultaneous anchorage with $\mathrm{Cu}-\mathrm{NPs}$ using $\mathrm{H}_{2} \mathrm{O}_{2}$ in conjunction with UV radiation. Furthermore, this hybrid material is easily dispersible in water. The anchorage of Cu-NPs to the CNT surface was studied as a function of the exposure time to microwave radiation. The produced materials were characterized by a combination of different spectroscopic and microscopy techniques. In order to assess their analytical and catalytic characteristics, the synthesized hybrid material was used for the carbohydrates reduction in aqueous media, in order to improve the detection of different carbohydrates including glucose, fructose and sucrose via CE.

\section{Experimental}

\subsection{Chemical methods}

Hydrogen peroxide 30\% was supplied by Panreac; ethanol, ferrocene $\left(\mathrm{FeCp}_{2}\right)$, toluene and aqueous solutions of $0.01 \mathrm{~N}$ sodium hydroxide volumetric standards were supplied by Sigma Aldrich; sodium hydroxide and $\mathrm{Cu}$ standard solutions of $1000 \mathrm{mg} / \mathrm{L}$ were purchased by Scharlau Chemie. Nitric acid (65\%) was supplied by Suprapur. Sucrose, D-glucose, and Dfructose standards, from Supelco Analytical, were prepared in aqueous solution at $500 \mathrm{ppm}$.

CNTs synthesized in the presence of minute amounts of oxygen (COx) were synthesized by chemical vapor deposition (CVD) as described by Botello-Mé ndez et al. [9]. Concentrations of $1 \%$ ethanol, $5 \%$ ferrocene and $94 \%$ toluene were selected because they provided highly crystalline CNT materials. For comparison purposes CNTs were also prepared under the same conditions but in the absence of alcohol; they are labelled as MWCNT in the text. As produced CNTs (COx) were then mixed with ethanol before the microwave treatment because it has been observed that the liquid reduces overheating (Joule effect). Subsequently, oxidized CNTs (oxdCOx) were prepared by a method reported in our previous publication by Martin et al. [10]. In particular, UV radiation (at $254 \mathrm{~nm}$ ) was applied to CNTs suspensions in water peroxide during 30 min. The suspensions were then filtered, washed and dried several times. This treatment permits the carboxyl-ation of CNTs increasing their dispersion ability when com-pared to pristine CNTs.

Fig. 1 shows the schematics depicting the experimental approach used in this work for anchoring Cu-NPs to CNTs, employing $\mathrm{Cu}\left(\mathrm{NO}_{3}\right)_{2}$ as a precursor and microwave radiation as the driver for promoting Cu-NPs encapsulation.

In a typical experiment, $100 \mathrm{mg}$ of oxdCOx were dispersed in a $0.1 \mathrm{~N}$ ethanol solution of $\mathrm{Cu}\left(\mathrm{NO}_{3}\right)_{2}$ which was dispersed ultrasonically during $5 \mathrm{~min}$. This dispersion was then introduced in a microwave oven for domestic use and then heated for different times. This resulted in the encapsulation of $\mathrm{Cu}-$ NPs by graphene-like layers (see below). The dispersions were

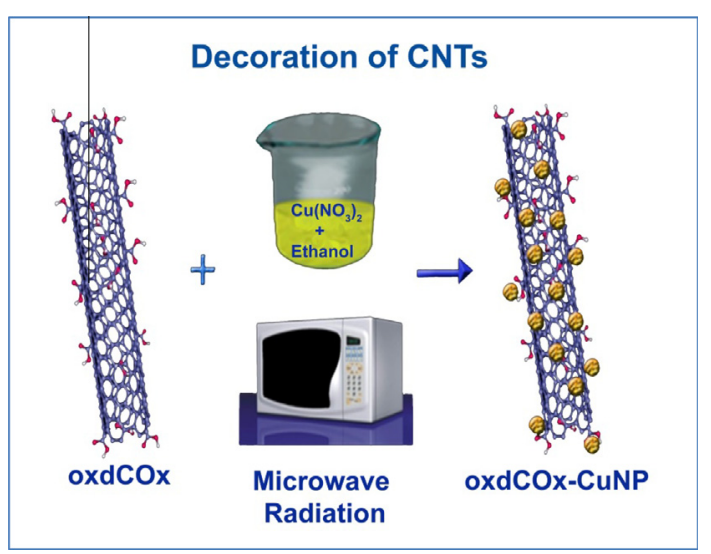

Fig. 1 - Schematic representation of the experimental approach used to anchor $\mathrm{Cu}-\mathrm{NP}$ on the CNTs surface. (A colour version of this figure can be viewed online.) 
then cleaned and filtered several times. Two treatment times were used: 1 and $2 \mathrm{~min}$. Sample Cu2min presented the best dispersibility so this study was concentrated on this sample.

\subsection{Instrumentation}

$\mathrm{X}$-ray photoelectron spectroscopy (XPS). Two XPS instruments were used: an Axis-Ultra (Kratos) and an Omicron. Surface chemical analysis was performed by $\mathrm{N}_{2}$ adsorption measurements at $77 \mathrm{~K}$ with a BELSORP-max (Bel Japan Inc). Samples were pressed into small pellets of $15 \mathrm{~mm}$ diameter, mounted on the sample holder and introduced into the chamber were they were degassed and vacuumed.

Titration. Carboxylic group concentration present within the CNT surface was measured using a Methrom 916 Ti-Touch Potentiometric compact titrator equipped with a Methrom 800 Dosino and a Unitrode $\mathrm{pH}$-meter. In a typical experiment, $10 \mathrm{mg}$ of $\mathrm{COx}$ or oxdCOx were added to $40 \mathrm{~mL}$ of Milli-Q water and then ultrasonicated for $1 \mathrm{~min}$ (VCX 500-750 of Sonics\&Materials, $750 \mathrm{~W}$ and $20 \mathrm{kHz}$ ). Subsequently, a $0.01 \mathrm{~N}$ $\mathrm{NaOH}$ solution was added at a rate of $1 \mathrm{~mL} / \mathrm{min}$ by $0.01 \mathrm{~mL}$ increments during mechanical stirring and the carboxylic acid concentration was determined from the equivalence point.

Transmission electron microscopy (TEM) analyses were carried out using a Zeiss EM-10C with a field emission gun operating at $60-100 \mathrm{kV}$. A suspension of CNTs in isopropanol was prepared ultrasonically and a drop was deposited onto lacey carbon grids.

High resolution transmission electron microscopy (HRTEM) and energy-dispersive (EDX) analyses were performed in a JEOL200 FX II microscope operated at $200 \mathrm{keV}$. TEM grids were prepared as described above taking the precaution of using $\mathrm{Ni}$ grids instead of $\mathrm{Cu}$ grids and identify the presence of $\mathrm{Cu}-\mathrm{NPs}$.

Thermogravimetric analysis (TGA) was performed in a Perkin Elmer $6000 \mathrm{STA}$ system heating from $50^{\circ} \mathrm{C}$ to $900^{\circ} \mathrm{C}$. Approximately $5 \mathrm{mg}$ of sample were heated in an open Pt crucible at a rate of $10^{\circ} \mathrm{C} / \mathrm{min}$ under an air flow $(20 \mathrm{~mL} / \mathrm{min})$.

Elemental analysis (EA) were carried out with a Perkin Elmer 1100B atomic absorption spectrophotometer, and by dispersive energy fluorescence X-ray (ED-XRF) in a SPECTRO XEPOS spectrometer in a He atmosphere with a Pd window, $50 \mathrm{~W} \mathrm{X-}$ ray excitation source and SDD detector. Determination of $\mathrm{Cu}$ was done digesting $4 \mathrm{mg}$ of samples in $\mathrm{HNO}_{3}$ during $15 \mathrm{~min}$ in a $600 \mathrm{~W}$ microwave chamber (Milestone Ethos D), and dissolved in $50 \mathrm{~mL}$ of Milli-Q water prior to measurements. Analysis of the as-synthesized CNTs had 3.3 wt\% Fe; once oxidized, iron content was reduced to $1.8 \mathrm{wt} \%$. Cu-NPs concentration in the hybrid CNTs materials yielded $0.27 \pm 0.01 \mathrm{mg} \mathrm{Cu} / \mathrm{g}$ and $0.41 \pm 0.03 \mathrm{mg} \mathrm{Cu} / \mathrm{g}$ for Cu1min and Cu2min, respectively.

Raman spectroscopy measurements were carried out in via Renishaw confocal microscope based Raman spectrometer using the $514.5 \mathrm{~nm}$ laser excitation. For each sample, various spectra were recorded in different places in order to verify the homogeneity of the sample.

Capillary electrophoresis (CE) experiments were carried out using a PA-800plus system from Beckman Coulter equipped with a DAD detector, set at $265 \mathrm{~nm}$. Silica capillaries of $75 \mu \mathrm{m}$ (inner diameter) were purchased from Polymicro Tech- nologies. They were cut in pieces with a total length of $58 \mathrm{~cm}$ (48 cm effective length). New capillaries were conditioned with $1 \mathrm{M}$ sodium hydroxide (10 $\mathrm{min}, 20 \mathrm{psi}$ ) and Milli-Q water (5 min, 20 psi). Fused silica capillaries were flushed with $0.1 \mathrm{M}$ $\mathrm{NaOH}$ during $10 \mathrm{~min}$, then, Milli-Q water was flowing during $3 \mathrm{~min}$, and finally $75 \mathrm{mM} \mathrm{NaOH}$ BGE during $7 \mathrm{~min}$, at 20 psi. Carbohydrate samples consisting of fructose, glucose and sucrose were diluted in Milli-Q water at $500 \mathrm{ppm}$, and they were then injected at 0.5 psi within $10 \mathrm{~s}$ period. Separations were performed at a constant voltage of $12 \mathrm{kV}$ and cassette temperature of $20^{\circ} \mathrm{C}$. COx, oxdCOx and Cu2min (from 0.003 to $0.008 \mathrm{mg} / \mathrm{mL}$ concentrations) were dispersed in $75 \mathrm{mM}$ $\mathrm{NaOH}$ using a $6 \mathrm{~mm}$ probe sonicator (model VCX-130-130 W from Biotech) during $10 \mathrm{~min}$ at $60 \mathrm{~Hz}$.

\section{Results and discussion}

\subsection{As produced and oxidized CNTs}

The functionalized surface of CNTs is crucial for achieving uniform dispersions in aqueous media. In this context, XPS analyses were used to characterize as produced and oxidized COx CNTs. XPS provides insightful information regarding the binding energies of carbon compounds. Fig. 2 plots deconvoluted XPS C1s and 01s spectra indicating the percentages of carboxylic (by $\mathrm{O}-\mathrm{C}=\mathrm{O}$ peak), alcoholic (by $\mathrm{C}-\mathrm{O}$ peak) and lactones (by $\mathrm{C}=\mathrm{O}$ peak) groups identified on the CNTs surface.

Deconvolution of the C1s peak for COx CNTs(left) shows a main peak centered at $284.5 \mathrm{eV}$, which is attributed to the graphitic structure ( $\mathrm{sp}^{2}$ hybridized carbon bonding) [24]. Peaks centered at 285.9, 287.3.0 and $289 \mathrm{eV}$ correspond to functional groups consisting of carbon atoms attached to different oxygen-containing moieties such as alcohol, lactones and carboxylic groups, respectively [37]. XPS analysis of the O1s peak (Fig. 2, COx) also confirms the presence of carboxylic groups on the CNTs surface (binding energy of $534.0 \mathrm{eV}$ ). Another peak was also identified at $530.7 \mathrm{eV}$, which corre-sponds to COx CNTs containing a large number of alcoholic groups (36.7\%), probably due to the presence of ethanol dur-ing synthesis. It is noteworthy that after the oxidation treat-ment there is a significant increase in the number of defects within the CNTs samples such as the increase of aliphatic groups, from $15.87 \%(\mathrm{COx})$ to $23.90 \%$ (oxdCOx). In addition, the concentration of carboxylic groups (see C1s plots) increased from $6.49 \%$ (COx) to $8.54 \%$ (oxdCOx) after oxidation; the nanotube dispersibility in aqueous media enhances significantly after oxidation (see below).

In order to confirm the presence of functional groups, acidbase titration experiments were also carried out to determine the concentrations of carboxylic groups on the CNTs surface (see Table S1 in Supplementary data). For exam-ple, the carboxylic acid deposited on the CNT surface increased up to $21 \%$ after the $\mathrm{H}_{2} \mathrm{O}_{2} / \mathrm{UV}$ oxidation treatment. The dispersion stability also significantly increased in time as illustrated in Fig. 3. Clearly, oxdCOx possess a better liquid stability and the suspension remained homogeneously dis-persed after 10 days. COx CNTs are only stable for few days after the dispersion preparation, whereas the pristine CNTs (pure carbon) settle down immediately after sonication. 

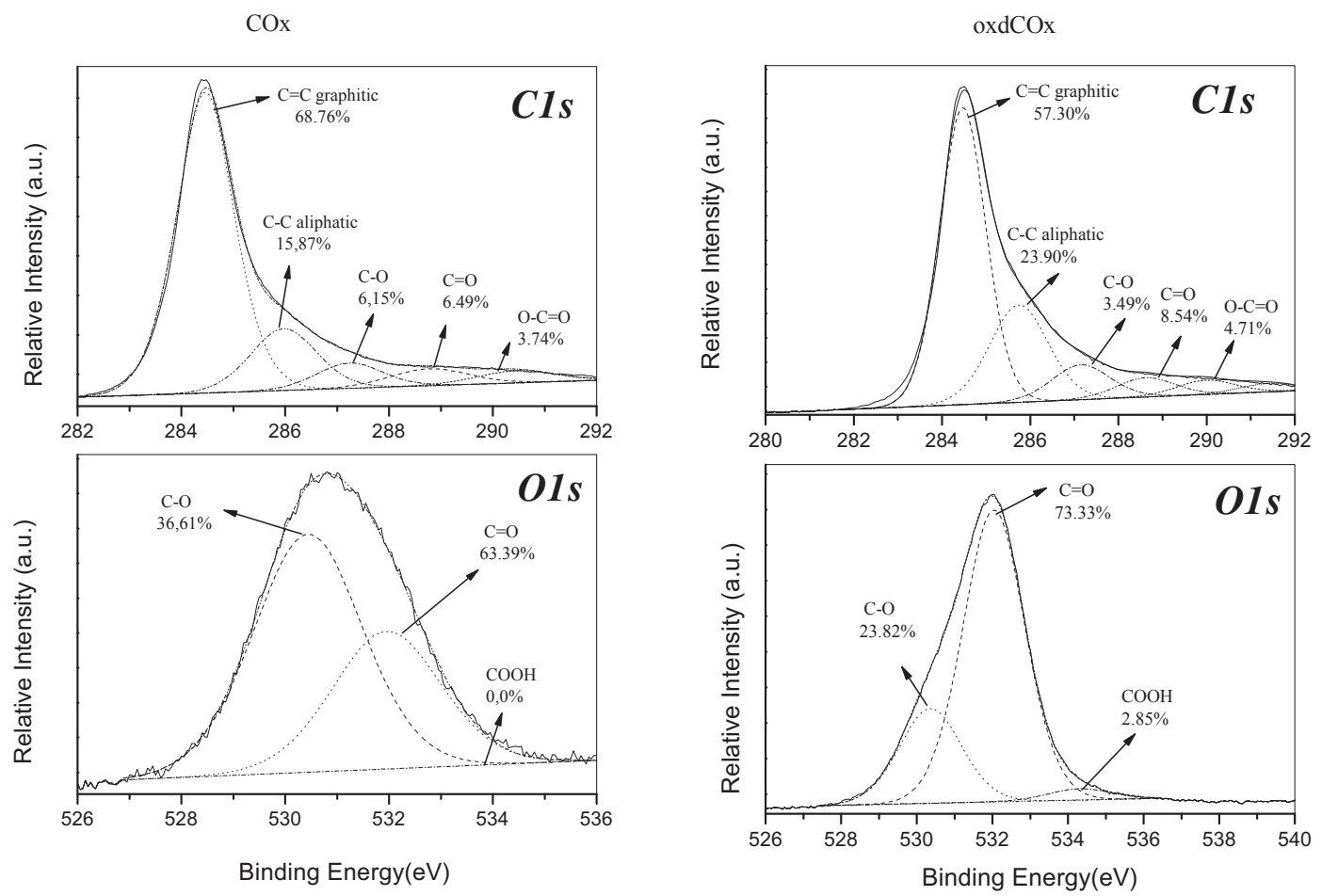

Fig. 2 - XPS spectra corresponding to the carbon (C1s) and oxygen (01s) binding energy of COx (left) and oxdCOx (right). The spectra were deconvoluted and the percentage $(\mathrm{m} / \mathrm{m})$ of the differential functional groups of alcohols $(C-O)$, lactones $(C=0)$ or carboxylic $(\mathrm{COOH})$ are indicated.

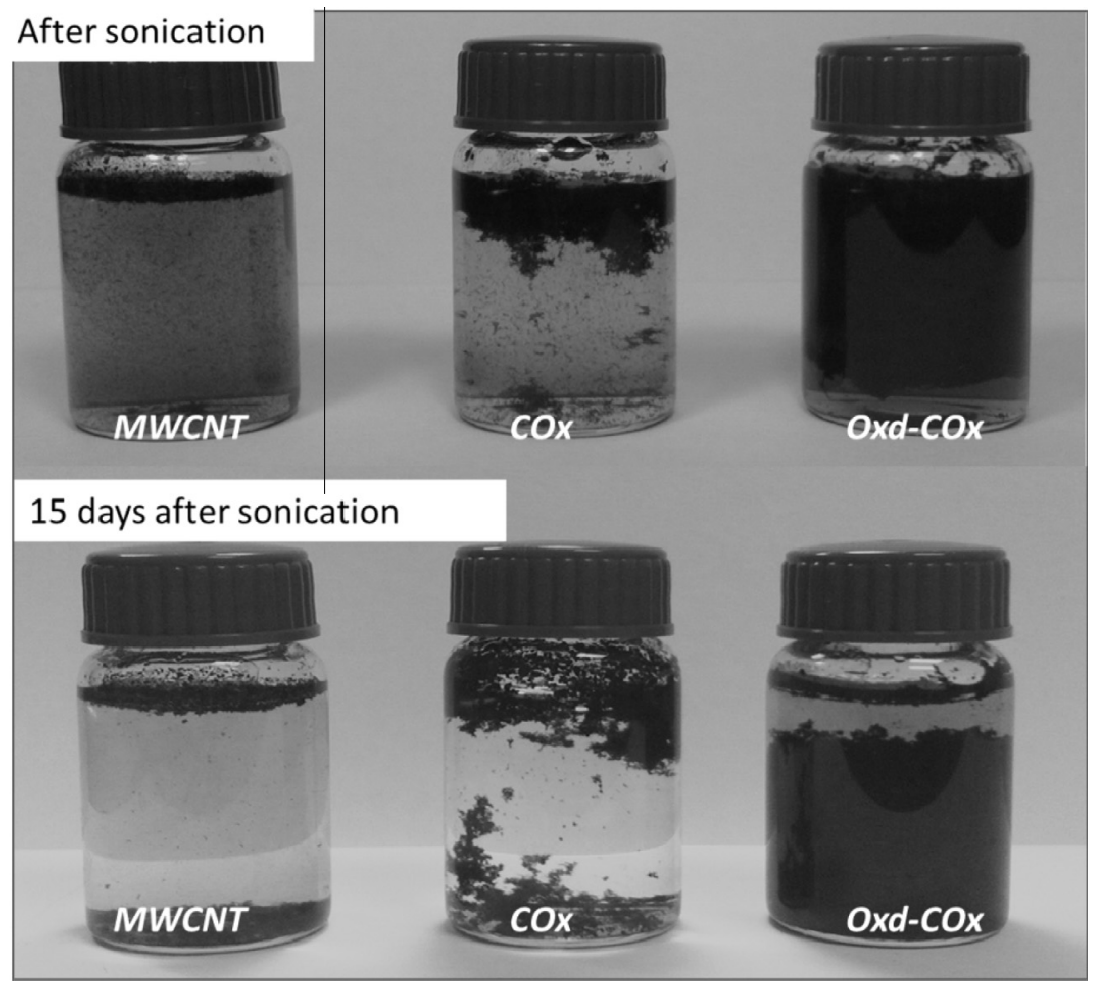

Fig. 3 - Photographs of aqueous CNT suspensions showing MWCNT, COx, and oxdCOx immediately after sonication and 15 days after sonication. 


\subsection{Encapsulation of Cu-NPs on carbon nanotubes}

Since oxdCOx displays a more stable dispersion when compared to COx CNTs, this sample was selected as support for depositing Cu-NP following the procedure described in the Section 2. TEM studies were used to confirm the anchorage of $\mathrm{Cu}-\mathrm{NP}$ on the surface of oxdCOx.

Fig. 4 shows TEM images of as synthesized CNT samples (COx), oxidized (oxdCOx) and the hybrid material after a $2 \mathrm{~min}$ microwave treatment. These images reveal a high amount of Fe-containing particles inside CNTs and also points that $\mathrm{Cu}-\mathrm{NPs}$ were deposited in a very low proportion on the surface of CNTs. No visible changes in the morphology of CNTs were observed after the Cu-NPs were anchored. This result is noteworthy since strong chemical oxidation of multiwalled CNTs usually damages the tubes surface [37]. According to our measurements, the Cu-NPs size distribution is centered at $10 \pm 2 \mathrm{~nm}$.

In order to confirm that the anchored particles on the surface of the tubes consisted of $\mathrm{Cu}$, EDX analyses were carried out using nickel TEM grids. In Fig. 5 the EDX spectrum of a spot (upper) is presented. The presence of $\mathrm{Cu}$ was clearly observed by observing the $\mathrm{Cu}-\mathrm{K}(8.6 \mathrm{keV})$ and $\mathrm{Cu}-\mathrm{L}(1.0 \mathrm{keV})$ transitions. In addition, $\mathrm{Fe}$ and $\mathrm{Ni}$ signals can be observed also; the signal from Fe could arise from the catalyst residues observed within the nanotubes (see Fig. 4) and the Ni signal must come from the grid background. Elemental analyses show that the $\mathrm{Fe}: \mathrm{Cu}$ atom ratio is $50: 1$, a very high ratio that makes difficult the determination of $\mathrm{Cu}$ by dispersive techniques. To prove that Fe particles are not at the surface of the CNTs a surface technique must be used.

The morphology of hybrid Cu2min and the way Cu-NPs anchored on CNTs were also studied by HRTEM. Fig. 6 shows an image of the Cu2min sample showing a Cu-NP with lattice fringes of ca. $0.208 \mathrm{~nm}$. This interlayer spacing is consistent with the (111) planes of $\mathrm{Cu}$ [29]. The (111) Fe plane also has a similar spacing but the fact that the examined particle is at the surface of a tube makes us to attribute this particle to $\mathrm{Cu}$.

To ascertain if Fe particles could be located not only within the tubes but also at their surface, XPS analysis was performed. Fig. 7 shows the results for the energy region corresponding to $\mathrm{Cu}$ for two samples, the oxidized COx and Cu2min. It can be clearly observed the signal from $\mathrm{Cu}$ $(933 \mathrm{keV})$ although its intensity is very low. No signal could be detected for Fe (see complete XPS spectrum in Supplementary data Fig. S3). Since XPS signals can only come from a very few atom layers from the surface, the absence of Fe signal is a clear indication that Fe particles are located within the tubes and that $\mathrm{Cu}$ particles are at the surface.

We also noted that Cu-NPs deposited on CNTs appear encapsulated by a few graphitic layers. This observation is noteworthy since it is not common to find Cu-NPs surrounded by graphitic layers after carrying out a chemical NP anchorage [24]. Similar results have been reported when depositing NPs under abrupt conditions [38]. Therefore, Cu-NPs appear to be firmly anchored to the surface of CNTs, thus making Cu-NPs highly stable in alkaline aqueous solutions used for the analytical studies described below (see Section 3.3).

The Raman spectra (see Fig. S1 in Supplementary data) were recorded for pristine COx, the oxidized CNTs (oxdCOx) and $\mathrm{Cu} 2 \mathrm{~min}$ samples. Each spectrum consists of three characteristic bands, namely the D-band located at ca. $1360 \mathrm{~cm}^{-1}$ (induced by the presence of amorphous carbon and defective sites embedded in $\mathrm{sp}^{2}$ hybridized lattices), the G-band located at ca. $1588 \mathrm{~cm}^{-1}$ (related to the in-plane tangential stretching $\operatorname{cox}$

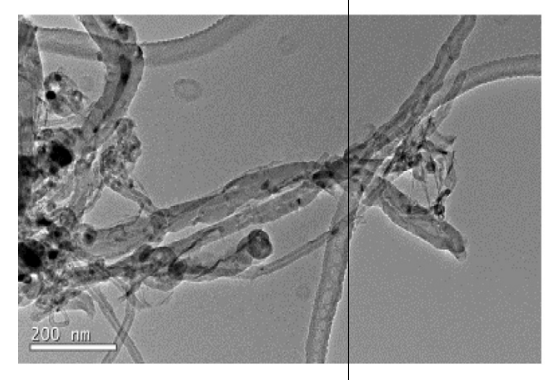

Cu2min

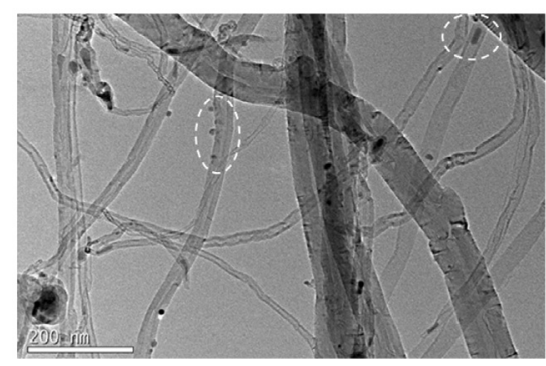

oxdCOx

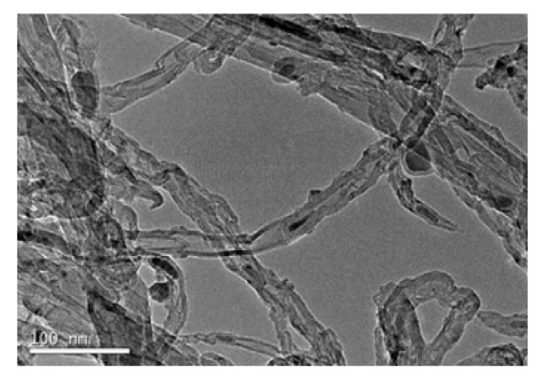

$\operatorname{oxdCOx}$

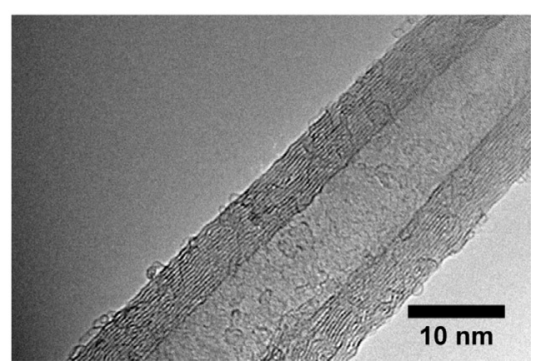

Fig. 4 - TEM images corresponding to COx and oxdCOx (upper) and Cu2min sample and oxdCOx magnification (bottom). For Cu2min some particles can be identified at the surface of a CNT; at the upper right, dotted line shows an iron particle inside a CNT and at the left, Cu particles in the surface of CNT. 


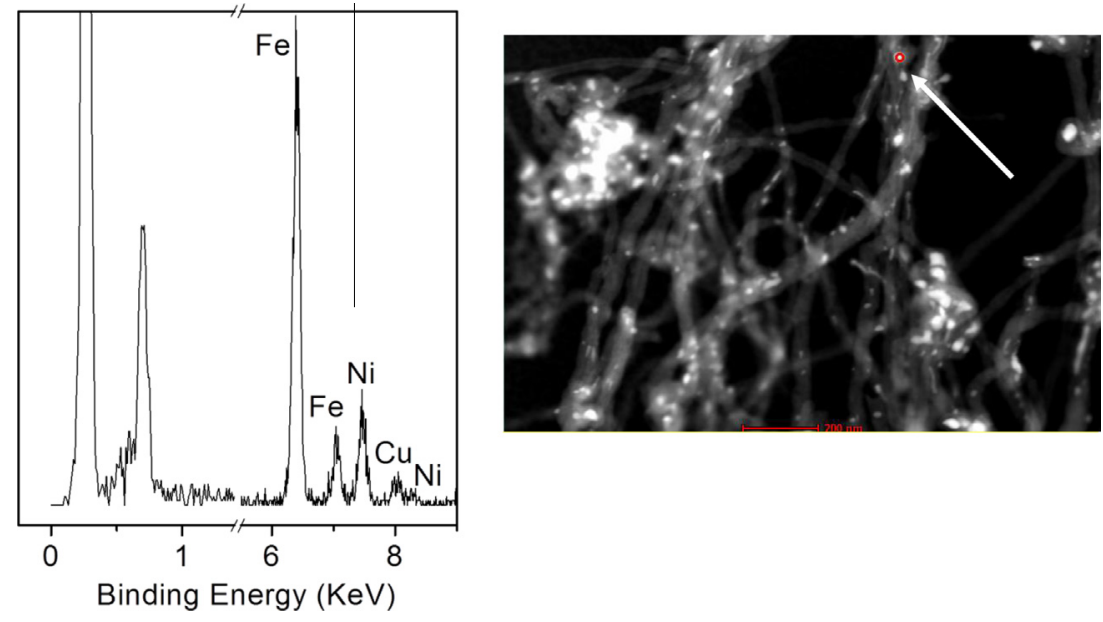

Fig. 5 - Spot beam EDX spectra (Ni grid) of Cu2min hybrid sample. Arrow shows the spot location. (A colour version of this figure can be viewed online.)

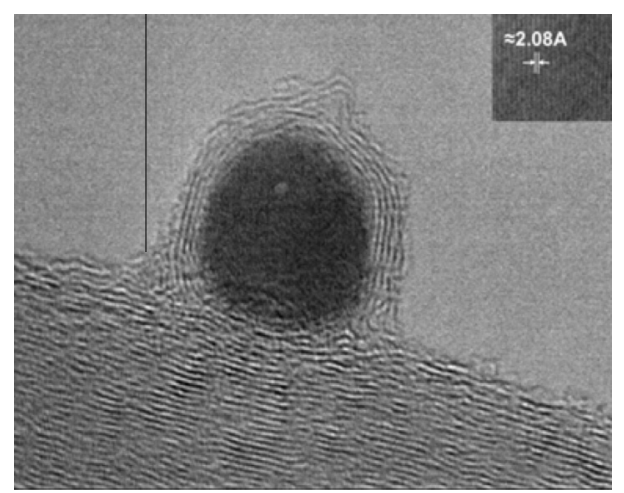

Fig. 6 - HRTEM images of a $10 \mathrm{~nm} \mathrm{Cu-NP} \mathrm{showing} \mathrm{an}$ interlayer distance of ca. $2.08 \AA$ that belong to the (111) plane of $\mathrm{Cu}$.

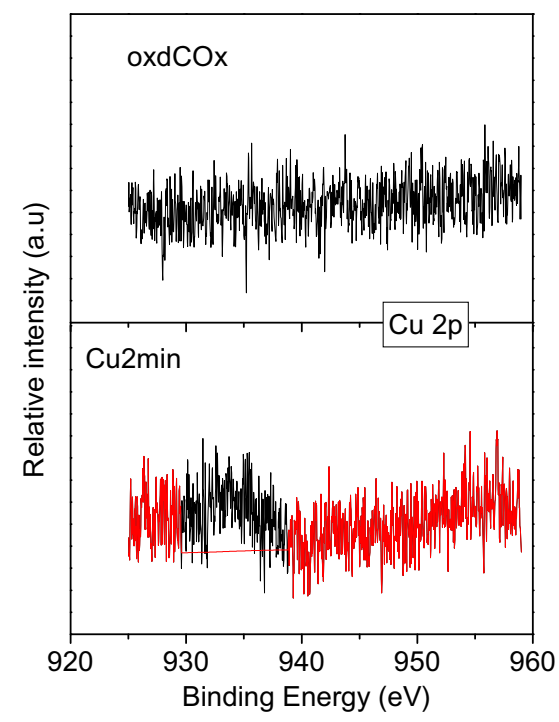

Fig. 7 - XPS spectra corresponding to the copper (Cu2p3/2) binding energy of oxdCOx (left) and oxdCOx-CuNP (right). (A colour version of this figure can be viewed online.)
Table 1 - Raman shifts for the D- $\left(\omega_{D}\right)$ and G- $\left(\omega_{G}\right)$ bands and the intensity ratio of the $D$ - and G-bands $\left(I_{D} / I_{G}\right)$. HT refers to heat treatment (see text for details).

\begin{tabular}{llll} 
Sample & $\omega_{\mathrm{D}}$ & $\omega_{\mathrm{G}}$ & $\mathrm{I}_{\mathrm{D}} / \mathrm{I}_{\mathrm{G}}$ \\
\hline COx & 1360.6 & 1588.2 & 0.51 \\
oxdCOx & 1358.9 & 1588.2 & 0.47 \\
Cu2min & 1360.8 & 1589.4 & 0.48 \\
oxdCOx HT & 1358.9 & 1589.5 & 0.53
\end{tabular}

carbon-carbon bonds in graphene sheets), and the 2D band centered at ca. $2707 \mathrm{~cm}^{-1}$. Raman shifts of the D- and G-bands and the intensity ratio of both bands $\left(I_{D} / I_{G}\right)$ are shown in Table 1.

The intensity ratio $I_{D} / I_{G}$ could be used to indicate the degree of crystallinity within $\mathrm{sp}^{2}$-hybridized carbon samples, that is, the smaller the number the more crystalline with fewer defects the sample becomes [39]. No significant variations in the spectral shape or Raman shifts were detected, in accordance with previous results [10], although a slight decrease in the $I_{D} / I_{G}$ ratio was observed as CNTs get oxidized and $\mathrm{Cu}-\mathrm{NPs}$ get anchored on the tube surfaces. These differences are within variations between different batches produced identically. Sample oxdCOx was treated at $500^{\circ} \mathrm{C}$ for $1 \mathrm{~h}$ under $\mathrm{N}_{2}$ to create a slightly more defective surface, as the slight increase in $I_{D} / I_{G}$ ratio reflects. This sample was used to confirm that catalytic activity was due to $\mathrm{Cu}-\mathrm{NPs}$ and not to surface interactions with the tubes (see Section 3.3).

Different structural forms of carbon can exhibit different oxidation behavior depending of the functionalities anchored to their surface [37]. For example, defects embedded in $\mathrm{sp}^{2}$ hybridized carbons tend to oxidize at around $500^{\circ} \mathrm{C}$ [40] due to their low activation energies, whereas nanocarbons exhibiting a high degree of crystallinity start to oxidize at a higher temperature, between 600 and $700{ }^{\circ} \mathrm{C}$ [41].

In our studies, TGA analysis (Fig. 8) revealed a decrease of the thermal stability of the oxidized sample (oxdCOx) when compared to the pristine nanotube sample [10]. In particular, 


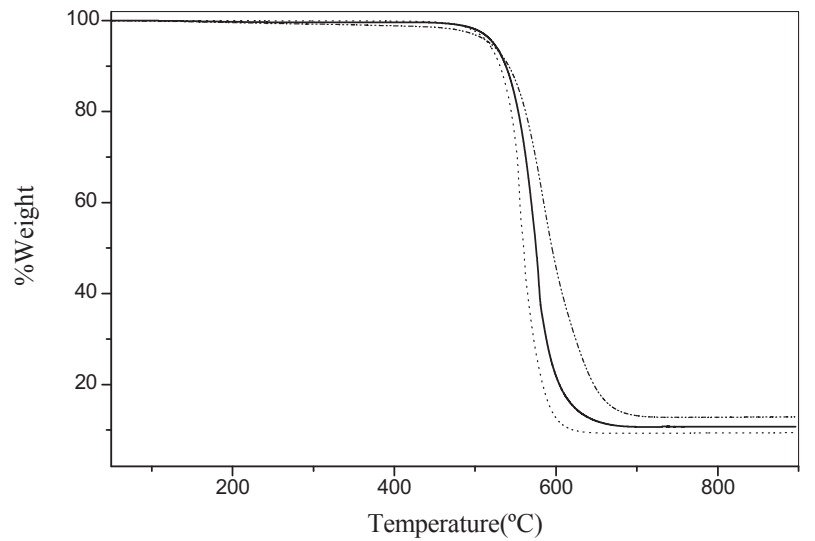

Fig. 8 - Thermogravimetric analysis (TGA) of different samples: COx (solid line); oxdCOx (dashed line); Cu2min (dashed dotted line).

the maximum oxidation rate for the COx sample appeared at $578^{\circ} \mathrm{C}$ whereas for the oxdCOx appeared at $550^{\circ} \mathrm{C}$. However, the Cu2min sample presented the best thermal stability since a maximum weight loss rate appeared at $587^{\circ} \mathrm{C}$. This stabilization effect created by $\mathrm{Cu}-\mathrm{NPs}$ could be due to the fact that $\mathrm{Cu}-\mathrm{NPs}$ are first deposited on defective sites, thus inhibiting oxidation sites within tubes. In addition, the microwave treatment which induced graphitization of carbon layers around the Cu-NPs, was also responsible for creating more crystalline graphitic surfaces; in agreement with the Raman observations described above.

\subsection{Analytical application: electrophoretic separation of carbohydrates}

Three carbohydrates (sucrose, glucose and fructose) were selected as analytes of interest for this work. However, their analysis in aqueous media by CE with UV-detection has been a challenge due to the lack of sensitive detection methods, because of the absence of charge at neutral $\mathrm{pH}$ and the absence of chromophore groups when they are underivatized [42]. In this context, some strategies have been proposed in order to overcome these problems [43]. Among those methods reported in the literature to separate and detect those underivatized carbohydrates, an aqueous BGE based on $\mathrm{NaOH}$ has been selected as a reference. This BGE favors the ionization of sugars due to their catalyzed hydrolysis under alkaline conditions [44,45]. Hydroxide ions initiate proton removal from the anomeric hydroxyl, followed by ring opening that produces their enolization, thus gaining enediolate anions with negative charge. The absorbance of the conjugated carbonyl groups will be higher than the original sugar, allowing its detection by UV-VIS at 265-270 nm [46,47], and being easily separated by CE as shown in Fig. 9. Moreover, from enols it is also possible to produce a large variety of oxidized products in an alkaline medium [45].

In this work, the separation of the carbohydrates using the conventional $\mathrm{NaOH}$ BGE has been compared to that produced by dispersions of oxdCOx (without Cu-NPs) and of the synthesized hybrid material $\mathrm{Cu} 2 \mathrm{~min}$ in $\mathrm{NaOH}$ as BGEs. oxdCOx and Cu2min samples were dispersed in $75 \mathrm{mM} \mathrm{NaOH}$ BGE in a

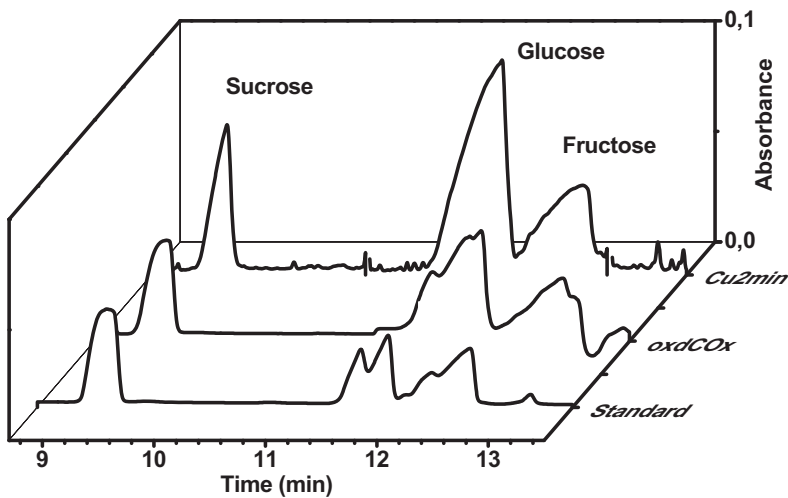

Fig. 9 - Sucrose, glucose, and fructose at 500 ppm were separated using as BGEs the standard $75 \mathrm{mM} \mathrm{NaOH}$ aqueous solution and the same aqueous solution containing $0.008 \mathrm{mg} / \mathrm{mL}$ of oxdCOx or Cu2min as indicated in the axis.

concentration range $0.003-0.008 \mathrm{mg} / \mathrm{mL}$. This concentration range was selected according to previously published ranges $[28,48,49]$, and Cu2min was chosen because it forms homogeneous dispersions, and because of the high amount of $\mathrm{Cu}$ efficiently anchored on the CNTs surface. The most relevant data, corresponding to the $0.008 \mathrm{mg} / \mathrm{mL}$ CNTs concentration are shown in Fig. 8.

The use of oxdCOx in the BGE leads to an improved sensitivity of glucose and fructose when compared to the BGE medium without CNTs. However, as observed in Fig. 8, the most impressive result appears with the Cu2min sample, which increases the sensitivity in the detection of these reducing carbohydrates by a factor of ca 5 . The sensitivity enhancement can be attributed to a heterogeneous catalysis effect of $\mathrm{Cu}$-NP on the glucose hydrolysis producing enolate anions in alkaline medium as fragmentation products [44]. Other synergistic effects on the oxidation of glucose in alkaline solution have been described in other combination of carbon nanostructures and CuNPs, including Cu-graphene $[26,50,29]$. The mechanism is not well understood although it has been proposed that the $\mathrm{Cu}$ (III)/Cu(II) pair may take part as an electron transfer mediator [50]. However, the presence of $\mathrm{Cu}$ ions is hard to accept in our system due to the encapsulation of Cu-NPs with graphitic layers. In order to verify that the sensitivity enhancement is only attributed to the presence of $\mathrm{Cu}-\mathrm{NPs}$ and not to the surface defects created during the oxidation process, we induced more defects on oxdCOx by heating the sample to $500{ }^{\circ} \mathrm{C}$ for $1 \mathrm{~h}$ in a $\mathrm{N}_{2}$ atmosphere. The high value of $I_{D} / I_{G}$ for this sample (see Table 1) confirms a higher defective surface but no significant changes were detected in the electropherogram (see fig. s2 in Supplementary data).

\section{Conclusions}

An easy approach has been successfully developed for the preparation of a hybrid system consisting of Cu-NPs encapsulated on the surface of CNTs. The results indicate that, under our experimental conditions, the amount of $\mathrm{Cu}$ is relatively low $(0.2-0.4 \mathrm{mg} \mathrm{Cu} / \mathrm{g}$, the size of $\mathrm{Cu}-\mathrm{NPs}$ is about $10 \mathrm{~nm}$ and are strongly anchored to the surface of oxidized CNTs. The 
dispersibility of the Cu-NPs hybrid material in aqueous solutions is high due to the presence of carboxylic groups formed on the surface of CNTs. We have used alkaline dispersions of these hybrid nanotubes as BGE for electrophoresis of a mixture of carbohydrates: sucrose, glucose and fructose. We have demonstrated that $0.008 \mathrm{mg} / \mathrm{mL}$ of $\mathrm{Cu} 2 \mathrm{~min}(0.41 \mathrm{mg} \mathrm{Cu} / \mathrm{g}$ sample) is enough to achieve a sensitivity enhancement of 5 times $(\mathrm{S} / \mathrm{N})$ in glucose when compared to media containing any nanostructure. The observed signal enhancement has been attributed to a heterogeneous catalysis of the carbohydrates fragmentation process due to the presence of $\mathrm{Cu}-\mathrm{NPs}$.

\section{Acknowledgements}

V. González, O. Martín and J. Baselga wish to thank Ministerio de Economía y Competitividad for funding through Grant MAT2010-17091. Authors also wish to thank to Sofía M. Vega for XPS measurements, Juan P. Fernández for TGA measurements and Lakshmy Pulickal Rajukumar for EDX measurements. C. Martín-Alberca thanks the University of Alcalá for his pre-doctoral Grant.

\section{R E F E R E N C E S}

[1] Iijima S. Helical microtubules of graphitic carbon. Nature 1991;354:56-8.

[2] Oberlin A, Endo M, Koyama T. Filamentous growth of carbon through benzene. J Cryst Growth 1976;32(3):335-49.

[3] Iijima S, Ichihashi T. Single-shell carbon nanotubes of 1-nm diameter. Nature 1993;363:603-5.

[4] Huang L, Jia Z, O'Brien S. Orientated assembly of singlewalled carbon nanotubes and applications. J Mater Chem 2007;17:3863-74.

[5] Nepal D, Balasubramanian S, Simonian AL, Davis VA. Strong antimicrobial coatings: single-walled carbon nanotubes armored with biopolymers. Nano Lett 2008;8(7):1896-901.

[6] Pumera M, Sanchez S, Ichinose I, Tang J. Electrochemical nanobiosensors. Sens. Actuator, B 2007;123:1195-205.

[7] Terrones M, Souza F, Rao A. Doped carbon nanotubes: synthesis, characterization and applications. In: Dresselhaus G, Dresselhaus MS, Jorio A, editors. Carbon

Nanotubes: Springer; 2008. Top. Appl. Phys.; vol. 111, pp. 531566.

[8] Cruz-Silva E, Lopez-Urias F, Muñoz-Sandoval E, Sumpter BG, Terrones H, Charlier J, et al. Electronic transport and mechanical properties of phosphorus and phosphorus nitrogen-doped carbon nanotubes. ACS Nano 2009;3(7):1913-21.

[9] Botello-Méndez A, Campos-Delgado J, Morelos-Gómez A, Romo-Herrera JM, Rodríguez AG, Navarro H, et al. Controlling the dimensions, reactivity and crystallinity of multiwalled carbon nanotubes using low ethanol concentrations. Chem Phys Lett 2008;453(1-3):55-6.

[10] Martin O, Gutierrez HR, Maroto-Valiente A, Terrones M, Blanco T, Baselga J. An efficient method for the carboxylation of few-wall carbon nanotubes with little damage to their sidewalls. Mater Chem Phys 2013;140:499-507.

[11] Georgakilas V, Gournis D, Tzitzios V, Pasquato L, Guldi DM, Prato M. Decorating carbon nanotubes with metal or semiconductor nanoparticles. J Mater Chem 2007;17:2679-94.

[12] Wildgoose GG, Banks CE. Metal nanoparticles and related materials supported on carbon nanotubes: methods and applications. Small 2006;2:192-3.

[13] Wang Z, Liu H, Chen L, Chou L, Wang X. Green and facile synthesis of carbon nanotube supported Pd nanoparticle catalysts and their application in the hydrogenation of nitrobenzene. J Mater Res 2013;28(10):1326-33.

[14] Sun T, Zhang Z, Xiao J, Chen C, Xiao F, Wang S, et al. Facile and green synthesis of palladium nanoparticles-graphenecarbon nanotube material with high catalytic activity. Sci Rep 2013;3:2527.

[15] Li H, Han L, Cooper-White J, Kim I. Palladium nanoparticles decorated carbon nanotubes: facile synthesis and their applications as highly efficient catalysts for the reduction of 4-nitrophenol. Green Chem 2012;14:586-91.

[16] Roy AK, Hsieh CT. Pulse microwave-assisted synthesis of Pt nanoparticles onto carbon nanotubes as electrocatalysts for proton exchange membrane fuel cells. Electrochim Acta 2013;87:63-72.

[17] Hsieh CT, Lin JY, Wei JL. Deposition and electrochemical activity of Pt-based bimetallic nanocatalysts on carbon nanotube electrodes. Int J Hydrogen Energy 2009;34:685-93.

[18] Hsieh CT, Chang YS, Yin KM. Pt-Sn nanoparticles decorated carbon nanotubes as electrocatalysts with enhanced catalytic activity. J Phys Chem C 2013;117:15478-86.

[19] Hsieh CT, Chen WY, Chen IL, Roy AK. Deposition and activity stability of Pt-Co catalysts on carbon nanotube-based electrodes prepared by microwave-assisted synthesis. J Power Sources 2012;199:94-102.

[20] He H, Gao C. Synthesis of Fe3O4/Pt Nanoparticles decorated carbon nanotubes and their use as magnetically recyclable catalysts. J Nanomater 2011;13(12-13):1-10.

[21] Li H, Cooper-White JJ. Hyperbranched polymer mediated fabrication of water soluble carbon nanotube-metal nanoparticle hybrids. Nanoscale 2013;5:2915-20.

[22] Fu Y, Zhang L, Chen G. Preparation of a carbon nanotubecopper nanoparticle hybrid by chemical reduction for use in the electrochemical sensing of carbohydrates. Carbon 2012;50:2563-70.

[23] Scarselli M, Scilletta C, Tombolini F, Castrucci P, Diociaiuti M, Casciardi S, et al. Multiwall carbon nanotubes decorated with copper nanoparticles: effect on the photocurrent response. J Phys Chem C 2009;113:5860-4.

[24] Wang X, Zhang F, Xia B, Zhu X, Chen J, Qiu S, et al. Controlled modification of multi-walled carbon nanotubes with $\mathrm{CuO}$, Cu2O and Cu nanoparticles. Solid State Sci 2009;11:655-9.

[25] Liao X, Lu S, Huang S. Preparation of copper/carbon sphere composites with excellent electrocatalytic activity toward glucose. Int J Electrochem Sci 2012;7:11274-80.

[26] Male KB, Hrapovic S, Liu Y, Wang D, Luong JHT. Electrochemical detection of carbohydrates using copper nanoparticles and carbon nanotubes. Anal Chim Acta 2004;516:35-41.

[27] Pop A, Manea F, Orha C, Motoc S, Ilinoiu E, Vaszilcsin N, et al. Copper-decorated carbon nanotubes-based composite electrodes for nonenzymatic detection of glucose. Nanoscale Res Lett 2012;7(1):266.

[28] Pauwels J, Van Schepdael A. Carbon nanotubes in capillary electrophoresis, capillary electrochromatography and microchip electrophoresis. Cent Eur J Chem 2012;10(3):785-801.

[29] Wang Z, Xia J, Qiang X, Xia Y, Shi G, Zhang F, et al. Polymerassisted in situ growth of copper nanoparticles on graphene 
surface for non-enzymatic electrochemical sensing of glucose. Int J Electrochem Sci 2013;8:6941-50.

[30] Ismaili H, Lagugné-Labarthet F, Workentin M. Covalently assembled gold nanoparticle-carbon nanotube hybrids via a photoinitiated carbene addition reaction. Chem Mater 2011;23(6):1519-25.

[31] Ma PC, Tang BZ, Kim JK. Effect of CNT decoration with silver nanoparticles on electrical conductivity of CNT-polymer composites. Carbon 2008;46:1497-505.

[32] Xie J, Wang S, Aryasomayajula L, Varadan VK. Platinum decorated carbon nanotubes for highly sensitive amperometric glucose sensing. Nanotechnology 2007;18:1-9.

[33] Fu Y, Zhang L, Chen G. Preparation of carbon nanotubecopper nanoparticle hybrid by chemical reduction for use in the electrochemical sensing of carbohydrates. Carbon 2012;50:2563-70.

[34] Leelaviwat N, Monchayapisut S, Poonjarernsilp C, Faungnawakij K, Kim KS, Charinpanitkul T. Microwaveinduced fabrication of cooper nanoparticle/carbon nanotubes hybrid material. Curr Appl Phys 2012;12:1575-9.

[35] Vazquez E, Prato M. Carbon nanotubes and microwaves: interactions, responses, and applications. ACS Nano 2009;3(12):3819-24.

[36] Imholt TJ, Dyke CA, Hasslacher B, Perez JM, Price DW, Roberts JA, et al. Nanotubes in microwave fields: light emission, intense heat, outgassing, and reconstruction. Chem Mater 2003;15:3969-70.

[37] Datsyuk V, Kalyva M, Papagelis K, Parthenios J, Tasis D, Siokou A, et al. Chemical oxidation of multiwalled carbon nanotubes. Carbon 2008;46:833-40.

[38] La Torre A, Rance GA, El Harfi J, Li J, Irvine DJ, Brown PD, et al. Transport and encapsulation of gold nanoparticles in carbon nanotubes. Nanoscale 2010;2:1006-10.

[39] Dillon AC, Yudasaka M, Dresselhaus MS. Employing Raman spectroscopy to qualitatively evaluate the purity of carbon single-wall nanotube materials. J Nanosci Nanotechnol 2004;4(7):691-703.
[40] Osswald S, Havel M, Gogotsi Y. Monitoring oxidation of multiwalled carbon nanotubes by Raman spectroscopy. J Raman Spectrosc 2007;38:728-36.

[41] Ajayan PM, Ebbesen TW, Ichihashi T, Iijima S, Tanigaki K, Hura $\mathrm{H}$. Opening carbon nanotubes with oxygen and implications for filling. Nature 1993;362:522-4.

[42] Soga T, Serwe M. Determination of carbohydrates in food samples by capillary electrophoresis with indirect UV detection. Food Chem 2000;69:339-44.

[43] Suzuki S, Honda S. A tabulated review of capillary electrophoresis of carbohydrates. Electrophoresis 1998;19:2539-60.

[44] Wrolstad RE. Reactions of sugars. In: Wrolstad RE, editor. Food carbohydrate chemistry. Oxford: Wiley-Blackwell; 2012. p. 35-47.

[45] Andreeva GG, Trusov SR. Liquid-phase catalytic oxidation of carbohydrates by oxygen: glucose oxidation in alkaline solutions in the presence of copper(II) complexes. Pharm Chem J 1995;29(8):557-9.

[46] Rovio S, Yli-Kauhaluoma J, Sirén H. Determination of neutral carbohydrates by CZE with direct UV detection. Electrophoresis 2007;28(17):3129-35.

[47] Sarazin C, Delaunay N, Costanza C, Eudes V, Mallet JM, Gareil P. New avenue for Mid-UV-range detection of underivatized carbohydrates and amino acids in capillary electrophoresis. Anal Chem 2011;83(19):7381-7.

[48] Xiong X, Ouyang J, Baeyens WRG, Delanghe JR, Shen X, Yang Y. Enhanced separation of purine and pyrimidine bases using carboxylic multiwalled carbon nanotubes as additive in capillary zone electrophoresis. Electrophoresis 2006;27:3243-53.

[49] Wang Z, Luo G, Chen J, Xiao S, Wang Y. Carbon nanotubes as separation carrier in capillary electrophoresis. Electrophoresis 2003;24:4181-8.

[50] Wu HX, Cao WM, Li Y, Liu G, Wen Y, Yang HF, et al. In situ growth of copper nanoparticles on multiwalled carbon nanotubes and their application as non-enzymatic glucose sensor materials. Electrochim Acta 2010;55:3734-40. 